\title{
BMJ Open Investigating the effectiveness of an online acceptance and commitment therapy (ACT) intervention versus a waiting list control condition on pain interference and quality of life in adults with chronic pain and multimorbidity: protocol for a randomised controlled trial
}

\author{
Brian W Slattery, ${ }^{1,2}$ Laura L O'Connor, ${ }^{1}$ Stephanie Haugh, ${ }^{1}$ Katie Barrett, ${ }^{1}$ \\ Kady Francis, ${ }^{1}$ Christopher P Dwyer, ${ }^{1}$ Siobhan O'Higgins, ${ }^{1}$ Line Caes, ${ }^{1}$ \\ Jonathan Egan, ${ }^{1}$ Brian E McGuire ${ }^{1}$
}

To cite: Slattery BW,

O'Connor LL, Haugh S, et al. Investigating the effectiveness of an online acceptance and commitment therapy (ACT) intervention versus a waiting list control condition on pain interference and quality of life in adults with chronic pain and multimorbidity: protocol for a randomised controlled trial. BMJ Open 2019;9:e012671. doi:10.1136/ bmjopen-2016-012671

- Prepublication history and additional material for this paper are available online. To view these files, please visit the journal online (http://dx.doi. org/10.1136/bmjopen-2016012671).

Received 25 April 2018 Revised 15 November 2018 Accepted 31 January 2019

Check for updates

(c) Author(s) (or their employer(s)) 2019. Re-use permitted under CC BY-NC. No commercial re-use. See rights and permissions. Published by BMJ.

For numbered affiliations see end of article.

Correspondence to Dr Brian W Slattery; brian.slattery@dcu.ie

\section{ABSTRACT}

Introduction Multimorbidity refers to the presence of two or more chronic health conditions within one person, where no one condition is primary. Research suggests that multimorbidity is highly correlated with chronic pain, which is pain lasting longer than 3 months. Psychotherapeutic interventions for people living with chronic illness have resulted in reduced symptom reporting and improved psychological well-being. There is a dearth of research, however, using online psychotherapy for people living with multimorbidity where chronic pain is a central condition. This study will compare the effectiveness of an online acceptance and commitment therapy (ACT) intervention with a waiting list control condition in terms of improving healthrelated quality of life (HRQLL) and reducing levels of pain interference in people with chronic pain and at least one other condition.

Methods and analysis 192 adult participants with nonmalignant pain that persists for at least 3 months and at least one other medically diagnosed condition will be randomised to one of two study conditions. The experimental group will undergo an eight-session internet-delivered ACT programme over an 8-week period. A waiting list group will be offered the ACT intervention after the 3-month follow-up period. HRQOL and pain interference will act as the primary outcomes. Data will be analysed using a linear mixed model and adjusted to account for demographic and clinical variables as necessary. A Study Within a Trial will be incorporated to examine the effect on recruitment and retention of showing participants an animated educational video.

Ethics and dissemination Ethical approval has been granted by the Research Ethics Committee of the National University of Ireland, Galway. Dissemination of results will be via peer reviewed journal articles and conference presentations. Trial registration number ISRCTN22343024.

\section{Strengths and limitations of this study}

- Interventions for people with chronic health problems tend to focus on one condition, and do not account for other symptoms or conditions a person may have.

- Research has shown that people with chronic pain typically have at least one other chronic health condition. To our knowledge this is the first randomised control trial to target the improvement of important health outcomes for people with chronic pain and multimorbidity.

- The aims of this study are novel and would provide useful information for both the applied and research communities, as well as potentially reducing pain interference and improving health-related quality of life for patients.

- This is the first study to adopt acceptance and commitment therapy (ACT) for multimorbidity and chronic pain and there may be issues with the adaptation. That said, the adaptation for the current study was supervised by a clinical psychologist who specialises in ACT and chronic pain and we do not envisage any issues, moreover, the efficacy of the programme as an intervention, is an empirical question and one which the study aims to answer.

\section{INTRODUCTION}

Background and rationale

Multimorbidity

Multimorbidity is defined 'as the co-existence of two or more chronic conditions, where one is not necessarily more central than the 
others'. (Boyd and Fortin, p453) ${ }^{1}$ Traditionally, medicine has taken a single disease approach to the management of chronic conditions. ${ }^{1}$ However, as Boyd and Fortin among others $^{23}$ suggest, such an approach to disease management is becoming increasingly untenable as greater numbers of people present with multiple conditions. In fact, as Violan $e t a t^{t}$ highlight, multimorbidity is now considered the norm rather than exception in primary care patients and some, including Salisbury, ${ }^{3}$ acknowledge that managing multimorbidity is the most important task facing health services in developed countries.

\section{Risk factors and prevalence}

Prevalence estimates of multimorbidity vary from country to country. For example, recent research in Australia suggests that $25.5 \%$ of the population live with multimorbidity, ${ }^{5}$ while in Scotland the prevalence of multimorbidity is reported to be $23.2 \%,{ }^{2}$ and in Ireland $45.3 \%$ of the population are reported to have multimorbidity. ${ }^{6}$ Indeed, prevalence rates of multimorbidity vary between subsets of different populations and are often found to be higher for those people who attend medical services. For example, Fortin and colleagues found that $90 \%$ patients in primary care in Canada had more than one condition, ${ }^{7}$ while in an Irish study, $66.2 \%$ of patients in primary care had multimorbidity. ${ }^{8}$

Although prevalence rates vary across populations and groups, it is clear that increasing numbers of people are developing multimorbidity. The rise in multimorbidity is due in part to improving technology, advancements in medicine and better health policies. ${ }^{9}$ In terms of risk factors however, research has shown that the most reliable predictor of multimorbidity is age. For example, one study found that the prevalence of two or more co-existing medical conditions in the age groups of 18-44 years, $45-64$ years, and 65 years and older were, $68 \%, 95 \%$ and $99 \%$ respectively. ${ }^{7}$ While multimorbidity is positively correlated with age, it must be noted that it is not only the burden of older generations, as Agborsangaya et $a l^{10}$ found that $70.2 \%$ of their sample under the age of 65 live with multimorbidity. Risk factors, including socioeconomic status, adverse childhood experiences, ${ }^{6}$ poor physical activity and risky health behaviours (eg, smoking) are also important contributors to multimorbidity. ${ }^{3}$

\section{Impact}

Living with multiple chronic conditions has debilitating physical, psychological, social and financial consequences for a person and their family. Specifically, multimorbidity increases the risk of engagement with healthcare providers (ie, hospitalisations), loss of physical functioning, depression, anxiety, polypharmacy and ultimately has an impact on a person's health related quality of life (HRQoL). ${ }^{11} 12$ HRQoL is a health outcome measure which is an indicator of an individual's overall well-being, and it is typically used to assess the effectiveness of interventions; it is therefore a predictor of treatment success and it is increasingly used to support 'allocation decisions in the healthcare sector'. ${ }^{13}$ Research has shown unequivocally that chronic disease has a negative impact on $\mathrm{HRQOL}^{1112}$; and it has been found that having multiple chronic conditions has an exponential impact on a person's wellbeing. ${ }^{13}$ Therefore, there is an interaction effect rather than a cumulative effect of multimorbid chronic conditions on HRQoL. ${ }^{13}$ Due to the increasing prevalence of multimorbidity and the burden of living longer with these conditions, the aim of improving HRQoL for people with multimorbidity has now become central to the focus of health practitioners. ${ }^{14}$

\section{Chronic pain and multimorbidity}

Chronic pain is defined as 'an unpleasant sensory and emotional experience associated with actual or potential tissue damage, or described by the patient in terms of such damage' that persists for a period in excess of 3 months. ${ }^{15}$ Chronic pain is a major public health problem that can have debilitating physical, emotional, psychological and financial consequences for those individuals living with it. ${ }^{16-18}$ Prevalence estimates for chronic pain vary, ${ }^{12-14} 1920$ however one recent study found that $35.5 \%$ of the Irish population were living with chronic pain. ${ }^{14}$

Chronic pain is highly correlated with multimorbidity, and consistently appears as one of the most common conditions in those identified as having multimorbidity. ${ }^{5}$ For example, in one Canadian study that examined the prevalence of disease-combinations, sixteen common disease pairs were identified, with chronic pain appearing in six of the combinations. Further, from the five most common disease triads identified in the same study, chronic pain was involved in three of these combinations. ${ }^{5}$ Boyd and Fortin ${ }^{1}$ noted that if a person had one chronic condition they were quite likely to also have another. Considering that over a third of the Irish population are reported to have chronic pain, and chronic pain is highly correlated with multimorbidity, it is important that research accounts for the relationship between the two.

Psychological interventions for multimorbidity and chronic pain Cognitive-behavioural therapy (CBT) is frequently used when a psychological treatment is required as part of a multidisciplinary rehabilitative process for people with chronic conditions and has been employed widely, for example, with people who live with chronic pain. ${ }^{8-13}$ Although CBT-based treatments are effective with many disorders, this is not the case for all conditions. ${ }^{21}$ As a result, research has investigated the effects of other psychotherapeutic approaches. ${ }^{22-24}$ Recently, acceptance and commitment therapy (ACT) has gained support for improving HRQoL in people living with chronic conditions. $^{23} 24$

Whereas CBT focuses on the reduction of symptom-related distress, ACT promotes 'psychological flexibility' (ie, the ability to engage with the present moment in a way that facilitates long term values). From the perspective of ACT, increasing psychological flexibility for a person with 
a chronic condition is a multistep process established by six core principles: acceptance of all experiences, both positive and negative; recognition of core personal values; committed action towards those values; psychological defusion; emphasis on the present moment; and sense of self as a context. ${ }^{25}$ ACT has been used as a psychological intervention to improve functioning and quality of life for people living with numerous chronic conditions, including depression ${ }^{26}$ tinnitus, ${ }^{27}$ diabetes,${ }^{28}$ cancer, ${ }^{29}$ post-traumatic stress ${ }^{30}$ and chronic pain. ${ }^{31}$

Indeed, a recent systematic review in the area of chronic pain ${ }^{32}$ found that ACT interventions were effective when compared with inactive treatment comparisons for improving physical functioning and reducing distress. Another systematic review of ACT in the context of chronic disease and long-term conditions found promising evidence to suggest ACT can be efficacious as a treatment in a number of ways, including disease self-management, despite the low number of high quality research studies using ACT as an intervention. ${ }^{33}$

\section{ACT online}

Traditionally, psychotherapeutic interventions, such as ACT, have been administered face-to-face when a client meets with a therapist. This face-to-face approach, however, is subject to numerous constraints including direct and indirect costs, high labour demands, long waiting lists, mobility and accessibility issues, and shortages in appropriately trained healthcare professionals. ${ }^{345}$ The provision of one-to-one therapy is therefore not currently feasible for wide-scale health interventions. To negate these limitations, researchers have begun to administer psychological interventions online. ${ }^{21}$ 36-41 These programmes provide standardised psychological treatment over the internet and are promising in their cost-effectiveness and accessibility, as results have shown them to be efficacious. ${ }^{41}$ For example, one recent randomised controlled trial examined the effectiveness of an online ACT intervention for people living with chronic pain. ${ }^{42}$ Participants were randomly assigned to an online treatment group for 7 weeks, or to a control group that participated in a moderated online discussion forum. Results showed that participants in the experimental group demonstrated increased activity engagement and willingness to experience pain and reductions were found on measures of pain-related distress, anxiety and depression. Furthermore, these improvements were maintained at a 6-month follow-up. ${ }^{42}$

\section{Objectives}

There is a large body of research that supports the use of psychotherapy and internet-delivered psychotherapy for people living with chronic pain. However, there is a dearth of research that attempts to improve HRQoL for people living with multimorbidity using any form of intervention, and there is no research that specifically examines the delivery of any form of psychotherapy to improve HRQoL and reduce pain interference for people living with multimorbidity where chronic pain is a feature. Moreover, ACT interventions for people living with chronic illnesses tend to be symptom-specific and target a particular disease; however, it is clear that a large proportion of people with one chronic illness live with one or more additional chronic conditions. In light of these issues, the proposed randomised control trial will examine the clinical effectiveness of an internet-delivered ACT intervention for people living with multimorbidity featuring chronic pain. It is hypothesised that people in the ACT treatment group will report significant improvements in pain interference, HRQoL, physical functioning, emotional functioning and rating of overall improvement, relative to a waiting list control group.

\section{Trial design}

The design is a single-blind randomised controlled trial comparing the effect of an internet-delivered ACT intervention with a waiting list control condition on HRQoL and pain interference (primary outcomes) for people with multimorbidity where chronic pain is one of their conditions. This protocol will be reported in accordance with the Standard Protocol Items: Recommendations for Interventional Trials guidelines (online supplementary file 1). ${ }^{43}$

\section{METHODS: PARTICIPANTS, INTERVENTIONS AND OUTCOMES Study setting}

The intervention is delivered in an online format and therefore participants can complete the intervention in their own homes. Study coordination and analysis will occur in the Centre for Pain Research at the National University of Ireland (NUI), Galway.

\section{Eligibility criteria}

Inclusion criteria: aged at least 18 years, the presence of two or more chronic conditions (including chronic pain) reported by the patient as having been diagnosed by a doctor, resident of Ireland, access to a computer/tablet and the internet, not currently undergoing any form of psychological treatment, sufficient competence in the English language (as determined by the participant) to complete the various elements of the study, informed consent is required. Exclusion criteria: severe cognitive impairment or psychiatric disorder that would interfere with the person's capacity to complete the study.

\section{Patient involvement}

This study is based on a previous programme conducted at the Centre for Pain Research at NUI Galway. ${ }^{33}$ Participant feedback (priorities, experiences and preferences), from that trial, and the pilot for this RCT were incorporated into the design of the intervention and the data collection. Patients will not be formally involved in recruitment for the study, but patient advocacy groups will be involved in promoting the study. We will communicate the results to participants by email when they are available. 


\section{Interventions}

\section{Experimental group}

The treatment protocol is almost identical to that used in a previous study by Hayes et al examining the clinical and cost effectiveness of an internet-delivered ACT intervention for people living with chronic pain. ${ }^{35}$ The intervention was derived from an ACT treatment manual specifically devised for people with chronic pain ${ }^{44}$ and adapted for online dissemination. Hayes et al utilised other resources ${ }^{45-47}$ and a team of healthcare professionals, including physiotherapists and clinical psychologists who specialise in chronic conditions and ACT treatments to revise the material so that it was modified accordingly and was suitable for online delivery. ${ }^{35}$ Thus, the online ACT treatment designed by Hayes et al is a robust adaptation and has been adopted for the purposes of the current research.

Appropriate changes to the content were made, so that the ACT programme designed by Hayes et al can be used in the current study for people with multiple conditions rather than for chronic pain only. The core concepts, homework, metaphors and mindfulness exercises, have for the most part, not been altered in any way. The only amendments necessary to the Hayes et al. ACT treatment programme were to alter the content that referred to symptoms of chronic pain specifically to instead refer to living with multimorbidity.

The experimental treatment will consist of eight sessions over an 8-week period and will be hosted on the NUI Galway, Centre for Pain Research Website. The programme will be delivered via an interactive online platform, and will consist of information, homework assignments, relevant metaphors and mindfulness exercises. The focus of this treatment protocol is on increasing psychological flexibility by developing acceptance, present-focused awareness and engagement in values-based action. An overview of the treatment is provided in table 1.

Over the course of the trial, participants in the experimental group will receive an automated weekly email reminder to complete each session. Adherence to the intervention will be monitored through login data. There is no clinician contact, and the programme is self-guided, with researchers only contacting participants (by phone or email) to prompt them should they fall behind. If a participant wishes to discontinue their involvement they will be withdrawn from the intervention and this will be reported as attrition. Technical questions and other queries are answered by email by the research team.

\section{Waiting list control group}

The waiting list control group will continue with their usual care, and will be contacted by the research team to complete questionnaires at 8 weeks postallocation and at 3 months follow-up. They will then be offered the opportunity to use the online ACT intervention following the 3-month follow-up assessment.

\section{Outcome measures}

\section{Demographic and clinical information}

Participants will be asked to provide details regarding age, gender, highest educational attainment, occupational status and relationship status as well as number and type of chronic conditions and duration of their conditions (including Body Mass Index) using a multimorbidity checklist. Some details about previous and current medical and alternative treatment will also be collected.

\section{Primary outcome measures}

Brief Pain Inventory-Short form

The Brief Pain Inventory (BPI)-Short form ${ }^{48}$ is a 9-item instrument that measures the interference and severity of a person's pain and the impact of their pain on their daily functioning, and is a standardised and widely used measurement tool for assessing chronic pain. The first eight items ask the person to provide demographic information, medicinal measures to alleviate pain symptoms, to identify locations of pain and to rate their pain severity from 1 to 10 (10 being the most severe pain) on a visual analogue scale over the past 24 hours. Item 9 is further divided into seven sub-items, examining interference with function. The subitems can then be grouped into those assessing physical functioning (ie, general activity, walking ability and working ability), those assessing psychological functioning (ie, mood, relations with other people and enjoyment of life) and one item investigating the extent to which pain affects sleep. The BPI has demonstrated good construct validity, adequate internal consistency, ${ }^{49}$ Cronbach's alpha, $\alpha=0.88$ and acceptable test-retest reliability. ${ }^{50}$

\section{Twelve-Item Short Form Survey}

The 12-Item Short Form Survey (SF-12) is a standardised instrument used as a measure of HRQoL. ${ }^{51}$ A shortened version of the SF-36, ${ }^{52}$ it reduces participant burden while still providing sub-scale scores across eight health domains (general health, physical functioning, emotional role limitation, physical role limitation, mental health, bodily pain, vitality and social functioning). It also produces two summary scores, a Mental Component Summary and a Physical Component Summary of HRQoL ${ }^{53}$ with lower scores on these scales representing lower quality of life. Both the overall score and the summary scores will be analysed, although the overall score will be the one of interest as primary outcome.

\section{Secondary outcome measures}

Acceptance and Action Questionnaire II

The original Acceptance and Action Questionnaire $(\mathrm{AAQ})^{54}$ has been used extensively with chronic conditions, ${ }^{29} 5556$ and the AAQ-II ${ }^{53}$ is widely used in measuring psychological inflexibility and experiential avoidance. The AAQ-II is a 7-item questionnaire, in which participants rate their responses to each item on a 1-7 scale in terms of how likely they are to accept or avoid aversive thoughts and feelings $(1=$ 'never true' and $7=$ ='always 
Table 1 Overview of the internet-delivered ACT intervention programme

\begin{tabular}{|c|c|c|}
\hline Week & Session & Summary of content \\
\hline 1 & Session 1 & $\begin{array}{l}\text { Introduction to the ACT programme and programme overview. } \\
\text { Review of treatment history and evaluate it in terms of how it has worked relative to the } \\
\text { participant's goals and expectations. } \\
\text { Review the interactions between thoughts, feelings and function, which often serve to make } \\
\text { each other worse (eg, become a 'vicious cycle'). } \\
\text { Introduce the idea that change is possible - not based on symptom reduction but on aiming } \\
\text { to alter function. } \\
\text { Introduction to mindfulness technique. } \\
\text { Homework assignment: check in with self daily and focus on activities. }\end{array}$ \\
\hline 2 & Session 2 & $\begin{array}{l}\text { Introduction to the concept of acceptance and how one's experience of their symptoms from } \\
\text { their various conditions may limit participation in valued activities. } \\
\text { Explanation of values. } \\
\text { Mindfulness explanation and debrief. } \\
\text { Homework assignment: Mindfulness practice daily. }\end{array}$ \\
\hline 4 & Session 4 & $\begin{array}{l}\text { Barriers to pursuing values. } \\
\text { Overcoming barriers. } \\
\text { Swamp metaphor - exercise exploring the possibility for values-based action even with } \\
\text { aversive experiences. Discussion on the concept of willingness and unwillingness to have } \\
\text { discomfort. } \\
\text { Body scan mindfulness exercise. }\end{array}$ \\
\hline 5 & Session 5 & $\begin{array}{l}\text { Goal setting exercise in line with three chosen values. } \\
\text { Discussion on fluctuating levels of high and low functioning and benefits of activity pacing in } \\
\text { order to achieve a more consistent level of activity from day to day. } \\
\text { Homework assignment: Record performance over the next week regarding carrying out } \\
\text { specific actions and pacing of activities and practice mindfulness every day. }\end{array}$ \\
\hline 7 & Session 7 & $\begin{array}{l}\text { Planning and action. } \\
\text { Willingness and committing to action. } \\
\text { Mindful walking exercise. } \\
\text { Homework assignment: commit yourself to action. }\end{array}$ \\
\hline 8 & Session 8 & $\begin{array}{l}\text { Emphasis on commitment to actions and values even when barriers exist and future planning } \\
\text { - this is a 'lifelong assignment'. } \\
\text { Preparation for relapses and setbacks. } \\
\text { End of programme. } \\
\text { Recap on topics covered throughout the programme. }\end{array}$ \\
\hline
\end{tabular}

ACT, acceptance and commitment therapy.

true'). The higher the participant scores overall, the greater the level of psychological flexibility. While there have been criticisms of the AAQ-II ${ }^{54}$ it has good content, construct, convergent and predictive validity, ${ }^{49}$ and satisfactory reliability.

Chronic Pain Acceptance Questionnaire-8

The Chronic Pain Acceptance Questionnaire-8 (CPAQ$8)^{58}$ - a shortened version of $\mathrm{CPAQ}^{53}$-is an 8 -item questionnaire and is rated on a scale from 0 to $6(0=$ 'never true' and $6=$ 'always true'). The questionnaire contains two subscales; pain willingness and activity engagement which are summed to indicate overall acceptance of pain. The CPAQ-8 has been validated in various populations, ${ }^{5960}$ has adequate to good reliability and consistency with Cronbach's alpha coefficients ranging from 0.69 to 0.86 and good test-retest reliability with an overall score correlation of $0.81 .^{61}$ 
Multimorbidity IIIness Perceptions Scale

The Multimorbidity Illness Perceptions Scale (MULTI$\mathrm{PleS})^{62}$ was developed to measure patient illness perceptions in the context of multimorbidity. Illness perceptions are a person's thoughts and feelings about their disease. The MULTIPleS is a 22-item questionnaire. The respondent indicates their level of agreement with each item on a Likert scale; the first 16 item scores range from 0 to 3, where ' 0 ' indicates that a person 'strongly disagrees' with an item and ' 3 ' indicates that a person 'strongly agrees' with an item, and the remaining six items range from 0 to 5 . Overall, the 22 items comprise five subscales; emotional representation, treatment burden, prioritising conditions, causal link and activity limitations. We will analyse the responses to the MULTIPleS across our three time points to assess for change overall or in these subscales. The MULTIPleS is relatively new - Gibbons $e t a t^{62}$ found that the scale provided a good fit to the Rasch model and demonstrated evidence of reliability and validity for each of the subscales.

\section{Patient Health Questionnaire-9}

The Patient Health Questionnaire-9 is a widely used measure of depression. ${ }^{63}$ Items relate to the criteria for depression in the Diagnostic and Statistical Manual of Mental Disorders (Version 5), and are scored on a 4-point Likert scale, ranging from 0 ('not at all') to 3 ('nearly every day'). Higher scores indicate the person meets more of the symptom criteria, scores above 10 indicates moderate depression and scores above 15 indicates a clinical case of moderately severe depression. It has been used and validated with chronic condition populations. ${ }^{17}$

\section{General Anxiety Disorder-7}

The General Anxiety Disorder 7 (GAD-7) a validated and standardised measure, will be used to measure anxiety. ${ }^{64}$ The 7-item questionnaire presents items relating to how often over the past couple of weeks a person has felt bothered by the seven DSM V criteria symptoms of generalised anxiety disorder. Items are scored on 4-point Likert scales ranging from 0 'not at all' to 3 'nearly every day'. A higher overall total score indicates greater symptom severity.

\section{Client Services Receipt Inventory}

The Client Services Receipt Inventory (CSRI) ${ }^{65}$ has been used widely in research examining the cost of chronic pain ${ }^{186667}$ and has been shown to be a valid measure of frequency of health service use. ${ }^{68}$ Medication and health service use will be measured at baseline, post-treatment and follow-up, using a modified version of the CSRI. As medication use will most likely vary throughout the trial, change in medication use (including prescribed and over-the-counter medications) will be examined in post-treatment analysis.

\section{Study timeline}

Interested participants will complete a screening questionnaire that will determine whether they satisfy the inclusion criteria, they will then complete another questionnaire that will capture demographic information and baseline data. Potential participants will receive a scripted phone call involving a further explanation and an opportunity to ask questions. They will then be randomised online to either the intervention or control group. Both groups will be asked to complete questionnaires at postintervention ( 8 weeks) and at follow-up (3 months). Participants in the waiting list control will then receive access to the programme. The process is outlined in the schematic diagram of participant flow (online supplementary file 2). As a point of note, a study within a trial will be conducted examining the effect of a short video on retention.

\section{Sample size}

In line with statistical convention and Cohen's recommendations, ${ }^{69}$ a sample size of 77 per group (total=154) will have the desired power of 0.8 , with a 0.025 alpha level to detect a medium effect size ( $d=0.50$ to 0.79 ) and both a significant clinical (ie, five point difference or one-half its SD $)^{68}$ and statistical difference in HRQoL between the experimental and control groups based on a two-tailed independent samples t-test. In accordance with the results of a previous study using online ACT for chronic pain, ${ }^{42}$ using an alpha value of 0.025 and a desired power of 0.8 , a total of 124 participants (62 per arm) are required to detect a medium difference in pain interference between the experimental and control groups. When calculating both sample sizes, an alpha value of 0.025 was used to account for multiple primary outcomes.

In order to detect both primary outcomes, the larger sample of 154 will be used. A recent study protocol with a similar design to the current study reported an expected $20 \%$ participant attrition rate between time 1 and time $3{ }^{42}$ To take account of this potential attrition rate, 192 participants will be recruited in the current study (96 per arm).

\section{Recruitment}

Participants will be recruited through advertisements about the study across a variety of contexts. Advertising will be done through websites that provide information on various chronic conditions typically found in an Irish context. Advertisements will also be posted in any relevant publications, forums or discussion boards where potentially interested parties are identified, and through social media and the website of the Centre for Pain Research at NUI Galway. Furthermore, information will be given to relevant healthcare professionals, groups and communities to disseminate about the trial as they see fit. Interested people will be directed to a website (Centre for Pain Research, at NUI Galway) where the trial is based, and encouraged to read additional information about the trial before they sign up. Potential participants will be told they can contact the research team should they have any questions or wish to clarify any information before they apply to participate. 


\section{METHODS: ASSIGNMENT OF INTERVENTIONS Allocation}

When a participant agrees to take part in the trial they will be randomly assigned to the intervention or waiting list control group using random permuted blocks to ensure groups are balanced. Randomisation will be performed using a custom-written script, administered from a password-secured server. As such, researchers do not hold influence in the allocation process.

\section{Blinding}

For initial analysis, the data analyst will be blinded to group allocation; however, because the trial delivers a psychological intervention, it is not possible to blind the participants to the groups they are in.

\section{METHODS: DATA COLLECTION, MANAGEMENT AND ANALYSIS Data collection methods}

All outcome measures administered at preintervention, postintervention and at 3-month follow-up are self-report and will be conducted online using the survey software Limesurvey (www.limesurvey.com). The participant attrition rate and any adverse events that may occur for the participants while they complete the intervention will also be recorded. Unless participants formally discontinue the study, attempts will be made to collect outcome data.

\section{Data management}

All study information will be collected online, using surveying software installed on a secure server managed by the research team at the Centre for Pain Research, School of Psychology, NUI Galway. The software and server are password protected and only two members (BEM and LLO) of the team hold the password. Non-identifiable information will be exported from the surveying software as needed, and shared only with the research team on secure computers based within the Centre for Pain Research at NUI Galway.

\section{Statistical methods}

Data will be analysed using the principles of intentionto-treat analysis. Date will be summarised by appropriate graphical (eg, box plots, labelled scatter plots and case profile plots) and numerical methods (eg, frequency counts, means, medians, SD and quartiles). The first primary outcome measure, the SF-12, produces scores on eight subscales and two summary scores which are transformed into a 0-100 scale. As such, the data from the SF-12 will be treated as a continuous variable, ${ }^{70}$ and the data from this measure over time (eg, baseline vs postintervention vs 6-month follow-up) will be analysed using a linear mixed model and adjusted accordingly to account for demographic and clinical variables as necessary. The second primary outcome, pain interference, measured by the BPI short-form, is scored by calculating the mean of seven different interference items; walking, work, mood, enjoyment of life, relations with others, sleep and general activity. The pain interference data are rated on a scale of $0-10$ and will be treated as a continuous variable and analysed as above. The secondary outcome measures will be treated as continuous variables also and analysed in a similar fashion. Each hypothesis will be tested using a two-tailed analysis at $\alpha=0.05$ level of significance, and missing data will be treated using multiple imputation analysis. A Bonferroni adjustment will be applied to results in order to account for the presence of multiple outcomes. All analyses will be completed using SPSS V.22 $2^{71}$ and Stata IC V.13 (StataCorp, 2013).

\section{METHODS: MONITORING \\ Data monitoring}

All study information will be collected online, using surveying software installed on a server managed by the research team. The software and server are password protected. Non-identifiable information will be exported from the surveying software as needed, and shared only with the research team.

\section{Adverse events}

Adverse events will be recorded. No harm is anticipated to arise from participating in this study. However, as with any psychotherapeutic intervention there is a slight chance that some content or their participation will cause distress to some participants. Participants will be made aware, should such an event arise, to contact a member of the research team who will refer them to appropriate support services. In addition, clinical staff within the Centre for Pain Research will be on hand to offer additional support if necessary.

\section{ETHICS AND DISSEMINATION \\ Protocol amendments}

In the event that amendments are made to the protocol, the trial registration will be updated. This study is based on a previously run study, as such significant changes are not anticipated, in the event that changes occur they will be communicated to participants via email.

\section{Consent}

Consent will be obtained electronically prior to enrolling in the study (online supplementary file 3 ). In addition, a phone call will offer participants the chance to formally withdraw before allocation.

\section{Confidentiality}

All study-related information will be stored securely at the Centre for Pain Research, School of Psychology, NUI Galway, where the research is taking place. Electronic data will only be accessible to the research team and will be stored on secure computers that are password-protected.

\section{Access to data}

Members of the research team will have access to the data during analysis. Anonymised participant level data 
and statistical code will also be available to researchers on request.

\section{Ancillary and post-trial care}

Given the nature of the study, it is not anticipated that participants will experience adverse effects. In the event that this occurs, participants are encouraged to contact the research team for further advice.

\section{Dissemination policy}

The findings of the trial will be submitted for publication in peer-reviewed journals and will be disseminated through conference presentations.

\author{
Author affiliations \\ ${ }^{1}$ Centre for Pain Research, School of Psychology, National University of Ireland, \\ Galway, Ireland \\ ${ }^{2}$ School of Nursing and Human Sciences, Dublin City University, Dublin, Dublin, \\ Ireland
}

Contributors $\mathrm{BS}, \mathrm{LLO}, \mathrm{KB}$ and $\mathrm{SH}$ are involved in the design, delivery and evaluation of the trial and also drafted the manuscript. KF, CD, SO, LC and JE were involved in the editing of the manuscript and will be involved in the evaluation of the trial. BEM contributed to the design of the intervention, supervises the delivery and evaluation of the study, and contributed to editing the manuscript.

Funding This work is supported by the Irish Health Research Board Research Leaders Award, grant reference (Brian McGuire, RLA/2013).

Competing interests None declared.

Patient consent for publication Not required.

Ethics approval Ethical approval has been granted by the National University of Ireland Galway Research Ethics Committee ref. 'NUI Galway Research Ethics Committee 16/JAN/01'.

Provenance and peer review Not commissioned; externally peer reviewed.

Open access This is an open access article distributed in accordance with the Creative Commons Attribution Non Commercial (CC BY-NC 4.0) license, which permits others to distribute, remix, adapt, build upon this work non-commercially, and license their derivative works on different terms, provided the original work is properly cited, appropriate credit is given, any changes made indicated, and the use is non-commercial. See: http://creativecommons.org/licenses/by-nc/4.0/.

\section{REFERENCES}

1. Boyd CM, Fortin M. Future of multimorbidity research: how should understanding of multimorbidity inform health system design? Public Health Rev 2010;32:451-74.

2. Barnett K, Mercer SW, Norbury M, et al. Epidemiology of multimorbidity and implications for health care, research, and medical education: a cross-sectional study. Lancet 2012;380:37-43.

3. Salisbury C. Multimorbidity: redesigning health care for people who use it. The Lancet 2012;380:7-9.

4. Violan C, Foguet-Boreu Q, Flores-Mateo G, et al. Prevalence, determinants and patterns of multimorbidity in primary care: a systematic review of observational studies. PLoS One 2014;9:e102149.

5. Britt HC, Harrison CM, Miller GC, et al. Prevalence and patterns of multimorbidity in Australia. Med J Aust 2008;189:72-7.

6. Sinnott C, Bradley CP. Multimorbidity or polypharmacy: two sides of the same coin? J Comorb 2015;5:29-31.

7. Fortin M, Bravo G, Hudon C, et al. Prevalence of multimorbidity among adults seen in family practice. Ann Fam Med 2005;3:223-8.

8. Glynn LG, Valderas JM, Healy P, et al. The prevalence of multimorbidity in primary care and its effect on health care utilization and cost. Fam Pract 2011;28:516-23.

9. Fortin M, Lapointe L, eds. Quality of life in primary care: a systematic Review. Hudon C Vanasse Ntetu AL Maltais Multimorb, 2004:9.

10. Agborsangaya $\mathrm{CB}, \mathrm{Ngwak}$ angnwi $\mathrm{E}$, Lahtinen $\mathrm{M}$, et al. Multimorbidity prevalence in the general population: the role of obesity in chronic disease clustering. BMC Public Health 2013;13:1161.
11. Lam CL, Lauder IJ. The impact of chronic diseases on the healthrelated quality of life (HRQOL) of Chinese patients in primary care. Fam Pract 2000;17:159-66.

12. Saarni SI, Härkänen T, Sintonen H, et al. The impact of 29 chronic conditions on health-related quality of life: a general population survey in Finland using 15D and EQ-5D. Qual Life Res 2006;15:1403-14.

13. Hunger $M$, Thorand $B$, Schunk $M$, et al. Multimorbidity and healthrelated quality of life in the older population: results from the German KORA-age study. Health Qual Life Outcomes 2011;9:53.

14. Hodek JM, Ruhe AK, Greiner W. [Relationship between healthrelated quality of life and multimorbidity]. Gesundheitswesen 2010;72:455-65.

15. IASP Terminology - IASP. https://www.iasp-pain.org/Education/ Content.aspx? ItemNumber=1698\&navltemNumber=576 (Accessed 24 Apr 2018).

16. Azevedo LF, Costa-Pereira A, Mendonça L, et al. Epidemiology of chronic pain: a population-based nationwide study on its prevalence, characteristics and associated disability in Portugal. J Pain Off J Am Pain Soc 2012;13:773-83.

17. Kroenke K, Outcalt S, Krebs E, et al. Association between anxiety, health-related quality of life and functional impairment in primary care patients with chronic pain. Gen Hosp Psychiatry 2013;35:359-65.

18. Raftery MN, Sarma K, Murphy AW, et al. Chronic pain in the Republic of Ireland--community prevalence, psychosocial profile and predictors of pain-related disability: results from the Prevalence, Impact and Cost of Chronic Pain (PRIME) study, part 1. Pain 2011;152:1096-103.

19. Mishra SI, Scherer RW, Geigle PM, et al. Exercise interventions on health-related quality of life for cancer survivors. In: Cochrane Database of Systematic Reviews. Chichester, UK: John Wiley \& Sons, Ltd, 2012.

20. Williamson DA, Rejeski J, Lang W, et al. Impact of a weight management program on health-related quality of life in overweight adults with type 2 diabetes. Arch Intern Med 2009;169:163.

21. Butler AC, Chapman JE, Forman EM, et al. The empirical status of cognitive-behavioral therapy: a review of meta-analyses. Clin Psychol Rev 2006;26:17-31.

22. Reibel DK, Greeson JM, Brainard GC, et al. Mindfulness-based stress reduction and health-related quality of life in a heterogeneous patient population. Gen Hosp Psychiatry 2001;23:183-92.

23. Lundgren T, Dahl J, Melin L, et al. Evaluation of acceptance and commitment therapy for drug refractory epilepsy: a randomized controlled trial in South Africa--a pilot study. Epilepsia 2006;47:2173-9.

24. Wicksell RK, Kemani M, Jensen K, et al. Acceptance and commitment therapy for fibromyalgia: a randomized controlled trial. Eur J Pain 2013;17:599-611.

25. Hayes SC, Strosahl K, Wilson KG. Acceptance and commitment therapy: an experiential approach to behavior change: Guilford Press, 1999.

26. Karlin BE, Walser RD, Yesavage J, et al. Effectiveness of acceptance and commitment therapy for depression: comparison among older and younger veterans. Aging Ment Health 2013;17:555-63.

27. Hesser H, Gustafsson T, Lundén $\mathrm{C}$, et al. A randomized controlled trial of Internet-delivered cognitive behavior therapy and acceptance and commitment therapy in the treatment of tinnitus. J Consult Clin Psychol 2012;80:649-61.

28. Gregg JA, Callaghan GM, Hayes SC, et al. Improving diabetes self-management through acceptance, mindfulness, and values: a randomized controlled trial. J Consult Clin Psychol 2007;75:336-43.

29. Feros DL, Lane L, Ciarrochi J, et al. Acceptance and Commitment Therapy (ACT) for improving the lives of cancer patients: a preliminary study. Psychooncology 2013;22:

30. Woidneck MR, Morrison KL, Twohig MP. Acceptance and commitment therapy for the treatment of posttraumatic stress among adolescents. Behav Modif 2014;38:451-76.

31. McCracken LM, Vowles KE. Acceptance and commitment therapy and mindfulness for chronic pain: model, process, and progress. Am Psychol 2014;69:178-87.

32. Hann KEJ, McCracken LM. A systematic review of randomized controlled trials of Acceptance and Commitment Therapy for adults with chronic pain: Outcome domains, design quality, and efficacy. $J$ Contextual Behav Sci 2014;3:217-27.

33. Graham CD, Gouick J, Krahé C, et al. A systematic review of the use of Acceptance and Commitment Therapy (ACT) in chronic disease and long-term conditions. Clin Psychol Rev 2016;46:46-58.

34. Dear BF, Gandy M, Karin E, et al. The Pain Course: a randomised controlled trial examining an internet-delivered pain management program when provided with different levels of clinician support. Pain 2015;156:1920-35. 
35. Hayes S, Hogan M, Dowd H, et al. Comparing the clinicaleffectiveness and cost-effectiveness of an internet-delivered Acceptance and Commitment Therapy (ACT) intervention with a waiting list control among adults with chronic pain: study protocol for a randomised controlled trial. BMJ Open 2014;4:e005092.

36. Andersson G, Cuijpers P. Internet-based and other computerized psychological treatments for adult depression: a meta-analysis. Cogn Behav Ther 2009;38:196-205.

37. Cuijpers P, Dekker J, Hollon SD, et al. Adding psychotherapy to pharmacotherapy in the treatment of depressive disorders in adults: a meta-analysis. J Clin Psychiatry 2009;70:1219-29.

38. Bender JL, Radhakrishnan A, Diorio C, et al. Can pain be managed through the Internet? A systematic review of randomized controlled trials. Pain 2011;152:1740-50.

39. Macea DD, Gajos K, Daglia Calil YA, et al. The efficacy of Web-based cognitive behavioral interventions for chronic pain: a systematic review and meta-analysis. J Pain 2010;11:917-29.

40. Spijkerman MP, Pots WT, Bohlmeijer ET. Effectiveness of online mindfulness-based interventions in improving mental health: A review and meta-analysis of randomised controlled trials. Clin Psychol Rev 2016:45:102-14.

41. McGuire BE, Henderson EM, McGrath PJ. Translating e-pain research into patient care. Pain 2017:158:190-3.

42. Buhrman M, Skoglund A, Husell J, et al. Guided internet-delivered acceptance and commitment therapy for chronic pain patients: a randomized controlled trial. Behav Res Ther 2013;51:307-15.

43. Chan AW, Tetzlaff JM, Gøtzsche PC, et al. SPIRIT 2013 explanation and elaboration: guidance for protocols of clinical trials. BMJ 2013;346:e7586.

44. Vowles KE, Wetherell JL, Sorrell JT. Targeting acceptance, mindfulness, and values-based action in chronic pain: findings of two preliminary trials of an outpatient group-based intervention. Cogn Behav Pract 2009;16:49-58.

45. Dahl J, Wilson KG, Nilsson A. Acceptance and commitment therapy and the treatment of persons at risk for long-term disability resulting from stress and pain symptoms: A preliminary randomized trial. Behav Ther 2004;35:785-801.

46. Hayes SC. Get Out of Your Mind and Into Your Life: the new acceptance and commitment therapy: New Harbinger Publications, 2005.

47. Dahl J, Luciano C, Wilson K. Acceptance and Commitment Therapy for Chronic Pain: New Harbinger Publications, 2005.

48. Cleeland CS, Ryan KM. Pain assessment: global use of the brief pain inventory. Ann Acad Med Singapore 1994;23:129-38.

49. Tan G, Jensen MP, Thornby JI, et al. Validation of the brief pain inventory for chronic nonmalignant pain. J Pain 2004;5:133-7.

50. Mendoza T, Mayne T, Rublee D, et al. Reliability and validity of a modified Brief Pain Inventory short form in patients with osteoarthritis. Eur J Pain 2006;10:353-61.

51. Ware J, Kosinski M, Keller SD. A 12-Item Short-Form Health Survey: construction of scales and preliminary tests of reliability and validity. Med Care 1996;34:220.

52. Ware JE, Sherbourne CD. The MOS 36-item short-form health survey (SF-36). I. Conceptual framework and item selection. Med Care 1992;30:473-83.
53. Bond FW, Hayes SC, Baer RA, et al. Preliminary psychometric properties of the Acceptance and Action Questionnaire-II: a revised measure of psychological inflexibility and experiential avoidance. Behav Ther 2011;42:676-88.

54. Francis AW, Dawson DL, Golijani-Moghaddam N. The development and validation of the Comprehensive assessment of Acceptance and Commitment Therapy processes (CompACT). J Contextual Behav Sci 2016;5:134-45

55. McCracken LM, Vowles KE, Eccleston C. Acceptance of chronic pain: component analysis and a revised assessment method. Pain 2004;107:159-66.

56. Westin V, Hayes SC, Andersson G. Is it the sound or your relationship to it? The role of acceptance in predicting tinnitus impact. Behav Res Ther 2008;46:1259-65.

57. Andrews G, Cuijpers P, Craske MG, et al. Computer therapy for the anxiety and depressive disorders is effective, acceptable and practical health care: a meta-analysis. PLoS One 2010;5:e13196.

58. Fish RA, McGuire B, Hogan M, et al. Validation of the chronic pain acceptance questionnaire (CPAQ) in an Internet sample and development and preliminary validation of the CPAQ-8. Pain 2010;149:435-43.

59. Rovner GS, Arestedt K, Gerdle B, et al. Psychometric properties of the 8-item Chronic Pain Acceptance Questionnaire (CPAQ-8) in a Swedish chronic pain cohort. J Rehabil Med 2014;46:73-80.

60. Baranoff J, Hanrahan SJ, Kapur D, et al. Validation of the chronic pain acceptance questionnaire-8 in an australian pain clinic sample. Int J Behav Med 2014;21:177-85.

61. Fish RA, Hogan MJ, Morrison TG, et al. Willing and able: a closer look at pain Willingness and Activity Engagement on the Chronic Pain Acceptance Questionnaire (CPAQ-8). J Pain 2013;14:233-45.

62. Gibbons CJ, Kenning C, Coventry PA, et al. Development of a multimorbidity illness perceptions scale (MULTIPIeS). PLoS One 2013;8:e81852.

63. Kroenke K, Spitzer RL. The PHQ-9: a new depression diagnostic and severity measure. Psychiatr Ann 2002;32:509-15.

64. Spitzer RL, Kroenke K, Williams JB, et al. A brief measure for assessing generalized anxiety disorder: the GAD-7. Arch Intern Med 2006;166:1092-7.

65. Beecham J, Knapp M. Costing psychiatric interventions. In: Thornicroft G, ed. Measuring Mental Health Needs. London, UK: Gaskell, 2001:200-24.

66. Critchley DJ, Ratcliffe J, Noonan S, et al. Effectiveness and costeffectiveness of three types of physiotherapy used to reduce chronic low back pain disability: a pragmatic randomized trial with economic evaluation. Spine 2007;32:1474-81.

67. Sleed M, Eccleston C, Beecham J, et al. The economic impact of chronic pain in adolescence: methodological considerations and a preliminary costs-of-illness study. Pain 2005;119:183-90.

68. Patel A, Rendu A, Moran P, et al. A comparison of two methods of collecting economic data in primary care. Fam Pract 2005;22:323-7.

69. Cohen J. A power primer. Psychol Bull 1992;112:155-9.

70. Busija L, Osborne RH, Nilsdotter A, et al. Magnitude and meaningfulness of change in SF-36 scores in four types of orthopedic surgery. Health Qual Life Outcomes 2008;6:55

71. IBM SPSS Statistics for Windows, Version 22.0. Armonk, NY, 2013. 